ARTICLE

https://doi.org/10.1038/s41467-019-12419-8

\title{
A Chlamydia pneumoniae adhesin induces phosphatidylserine exposure on host cells
}

Jan N. Galle (1) 1, Tim Fechtner ${ }^{1}$, Thorsten Eierhoff ${ }^{2,3,4,5}$, Winfried Römer (10 ${ }^{3,4}$ \& Johannes H. Hegemann (D) ${ }^{1 \star}$

In mammalian cells, the internal and external leaflets of the plasma membrane (PM) possess different phospholipids. Phosphatidylserine (PS) is normally confined to the inner (cytoplasmic) membrane leaflet. Here we report that the adhesin CPnO473 of the human pathogenic bacterium Chlamydia pneumoniae ( $\mathrm{Cpn}$ ) binds to the PM of human cells and induces PS externalization but unexpectedly not apoptosis. PS externalization is increased in human cells exposed to infectious Cpn cells expressing increased CPnO473 and reduced in exposure to $\mathrm{Cpn}$ expressing decreased $\mathrm{CPnO} 473$. CPnO473 binds specifically to synthetic membranes carrying PS and stimulates pore formation. Asymmetric giant unilamellar vesicles (GUVs) in which PS is restricted to the inner leaflet reveal that $\mathrm{CPnO} 473$ induces PS externalization in the absence of other proteins. Thus our identification of CPnO473 as a bacterial PS translocator capable of specific and apoptosis-independent PS externalization during infection extends the spectrum of mechanisms intracellular pathogens use to enter host cells.

\footnotetext{
${ }^{1}$ Lehrstuhl für Funktionelle Genomforschung der Mikroorganismen, Heinrich-Heine-University Düsseldorf, Universitätsstraße 1, 40225 Düsseldorf, Germany ${ }^{2}$ Lehrstuhl für Molekulare Evolution, Heinrich-Heine-University Düsseldorf, Universitätsstraße 1, 40225 Düsseldorf, Germany. ${ }^{3}$ Faculty of Biology, AlbertLudwigs-University Freiburg, Schänzlestraße 1, 79104 Freiburg, Germany. ${ }^{4}$ Signalling Research Centres BIOSS and CIBSS, Albert-Ludwigs-University Freiburg, Schänzlestraße 18, 79104 Freiburg, Germany. ${ }^{5}$ Department of Vascular and Endovascular Surgery, University Hospital Münster, Albert Schweitzer Campus 1, 48149 Münster, Germany. *email: johannes.hegemann@hhu.de
} 
T he mammalian plasma membrane (PM) exhibits a distinct phospholipid (PL) asymmetry, which is established and maintained by specific ATP-driven lipid translocators (flippases, floppases) ${ }^{1,2}$. Transmembrane movement of phosphatidylserine (PS) from the inner to the outer leaflet of the PM is called externalization, a process executed by scramblases, which occurs in apoptotic cells (triggering their removal by phagocytes) and during platelet activation ${ }^{3,4}$. However, the precise molecular mechanisms that induce or modulate PS externalization are poorly understood.

Chlamydia pneumoniae is an important obligate intracellular Gram-negative bacterial pathogen that infects the upper and lower respiratory tract and is implicated in a wide range of chronic diseases including atherosclerosis and Alzheimer's disease $^{5}$. The infectious chlamydial elementary body (EB) gains entry to host cells by receptor-mediated endocytosis and replicates in a membrane-bound compartment called an inclusion ${ }^{6-9}$. We previously identified CPn0473 as a Cpn-specific adhesin involved in $\mathrm{EB}$ internalization and showed that recombinant CPn0473 (rCpn0473) dose dependently enhances EB entry into host cells, thus promoting infection ${ }^{10}$.

In this manuscript, we demonstrate that the chlamydial adhesin CPn0473 binds negatively charged PLs and its C terminus inserts into the host-cell PM. CPn0473 acts as a PL translocator, which transports host-cell PS from the inner cytosolic leaflet of the PM to the extracellular leaflet. CPn0473-mediated PS externalization does not induce apoptosis. CPn0473 binding to synthetic asymmetric membranes results in directional PS translocation from the inner (PS-high) to the outer (PS-low) leaflet. PS translocation via $\mathrm{CPn} 0473$ facilitates uptake of the infectious chlamydial EB by the host cell.

\section{Results}

CPn0473 associates with negatively charged PLs. To investigate the protein's function further, we screened for interaction partners and found that recombinant $\mathrm{rCPn} 0473$ binds strongly to liposomes in pull-down experiments (Fig. 1a). Assays performed with nitrocellulose strips spotted with different lipids indicated that rCPn0473 binds to various negatively charged PLs (Supplementary Fig. 1a). Using giant unilamellar vesicles (GUVs) made of defined lipid mixtures, we confirmed the specificity of rCPn0473 for negatively charged PLs, and verified that rCPn0473 did not bind GUVs consisting of phosphatidylcholine (DOPC). Strikingly, rCPn0473 showed a markedly higher affinity for PS (DOPS) than for phosphatidic acid (DOPA, PA), whereas phosphatidylinositol 4,5-bisphosphate (PI(4,5)P2) was not recognized (Fig. 1b, c). Raising the fraction of PS in the lipid mixture from 5 to $20 \mathrm{~mol} \%$ enhanced binding of rCPn0473 (Fig. 1d), comparable to the positive control rLactC2, which exclusively binds $\mathrm{PS}^{11}$ (Supplementary Fig. 1a-c, Fig. 1e). As invasion by Cpn EBs occurs via cholesterol-rich membrane domains ${ }^{10,12}$, we also tested GUV membranes supplemented with cholesterol, which indeed increased binding to GUVs generally. Interestingly, a rCPn0473 variant lacking the domain required for binding to human cells (BD, aa $307-356)^{10}$ adhered to GUVs, whereas removal of residues 1-171 (essential for stimulating EB internalization ${ }^{10}$ ) abrogated the interaction. Conversely, fusion of the first 176 aa of CPn0473 to OmcB enabled the latter to adhere to PS-containing GUVs (Fig. 1e, f, Supplementary Fig. 1c).

Efficient chlamydial infection depends on recognition of PS. To assess the relevance of PS for infection by CPn, we infected the PS-deficient mutant cell line CHO-PSA $3^{13}$. We observed a significant reduction in Cpn infection relative to wild-type CHO$\mathrm{K} 1$ cells, indicating that PS is important for infection (Fig. 2a).
Next, we tested whether the rCPn0473-mediated boost in EB internalization depends on host-cell $\mathrm{PS}^{10}$. CHO-K1 cells preexposed to rCPn0473 indeed exhibited an increase in internalized EBs compared with bovine albumin (BSA)-treated cells, but no effect was observed in the PS-deficient line pre-treated with rCPn0473 (Fig. 2b). Importantly, no significant difference in rCPn0473 binding was noted between K1 and PSA3 cells (Supplementary Fig. 1d). Overall, these data indicate that CPn0473 binds to PS, and that PS is essential for the rCPn0473-mediated boost in both EB internalization and infectivity.

Interestingly PS, which under homeostatic conditions is restricted to the inner leaflet of the $\mathrm{PM}^{14}$, is externalized upon infection with $C p n^{15}$. Indeed, when we assayed $C p n$-infected cells for PS externalization with fluorescent annexin- $\mathrm{V}^{16}$, a signal was observed surrounding adhering EBs as early as 5 min post infection (pi), whereas heat-inactivated CPn EBs did not induce fluorescence (Fig. 2c). At $60 \mathrm{~min} \mathrm{pi,} 83 \pm 9 \%$ of Cpn EBs were annexin-V-positive $( \pm=$ standard deviation (SD) of mean data, Fig. 2d). Interestingly, the association of adhering Cpn EBs with annexin- $\mathrm{V}$ signals was species-specific, as only $15 \pm 3 \%$ of $C$. trachomatis EBs of serovar E and only $9 \pm 1 \%$ of serovar LGV exhibited annexin-V fluorescence (Fig. 2d). To confirm this result, we assayed PS exposure by $C p n$ using rLactC2, and again detected externalized PS at EB entry sites. Interestingly, both Cpn and rLactC2 signals accumulated in cholera toxin (CTxB)positive, cholesterol-enriched membrane domains ${ }^{17}$ (Supplementary Fig. 2a).

CPn0473 induces PS externalization. As CPn0473 is specific to $C p n$, and only Cpn EBs showed massive PS externalization, we tested whether rCPn0473 itself could induce PS translocation by incubating HEp-2 cells with rCPn0473. Indeed, we observed PS externalization at the binding site of rCPn0473 in a time- and dose-dependent manner visualized by Annexin- $\mathrm{V}$ and rLactC2fluorescence intensity (FITC), whereas a triple-AAA mutant version of rLactC2-FITC that does not bind PS failed to stain rCPn0473-bound cells (Fig. 3a-c, Supplementary Fig. 2b). Importantly, when using the FITC-labeled $\mathrm{PI}(4,5) \mathrm{P}_{2}$-binding domain of PLC $\gamma$ and the PI(3)P-binding FYVE domain of the Hgs (Supplementary Fig. 1a, e, f), we did not detect any externalization of phosphatidylinositol 4,5-bisphosphate $\left(\mathrm{PI}(4,5) \mathrm{P}_{2}\right)$ and phosphatidylinositol 3-phosphate (PI(3)P), two other negatively charged PLs that are normally restricted to the inner leaflet of the PM, to the host-cell surface upon the addition of rCPn0473 to HEp-2 cells (Supplementary Fig. 2c), evidencing specificity of rCPn0473 for PS. The extent of annexin fluorescence correlated with the amount of rCPn0473 used, and increased over time, first becoming detectable after $15 \mathrm{~min}$ of incubation (Fig. 3b, c, Supplementary Fig. 3a). As calcium entry might trigger cellular PS exposure, we tested different calcium concentrations in the medium of HEp-2 cells. Notably, the level of rCpn0473-induced PS externalization was not enhanced by elevated extracellular calcium levels (Fig. 3b).

PS externalization mediated by $\mathrm{CPn} 0473$ is non-apoptotic. Further characterization of the CPn0473-induced PS externalization process revealed that it is sensitive to cholesterol depletion by methyl-beta-cyclodextrin $(\mathrm{M} \beta \mathrm{CD})$ and did not rely on an intact cortical microtubule and actin meshwork as proofed by nocodazole or cytochalasin D treatment (Fig. 3d, Supplementary Fig. 3b-d). PS externalization is a hallmark for apoptosis, which is triggered by the proteolytic activation of caspase- 3 and inactivation of PARP. Importantly, no cleavage of pro-caspase-3 or PARP could be detected in cells exposed to rCPn0473 or in Cpn-infected cells, in contrast to staurosporine-treated, apoptotic cells (Fig. 3e). 
a

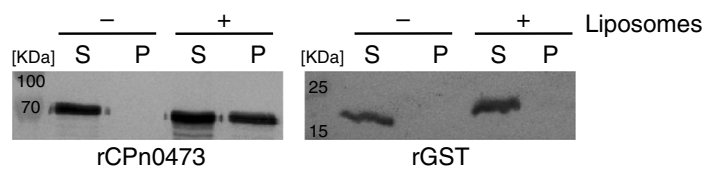

b

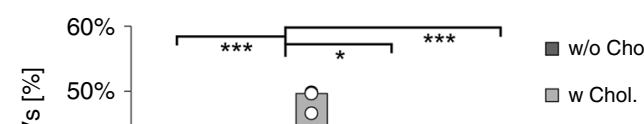

0
0
0
0
2
0
0
0
0
0
0
0
0
0
0
0

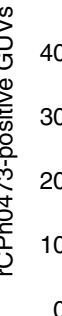

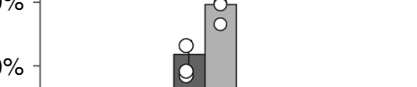

c
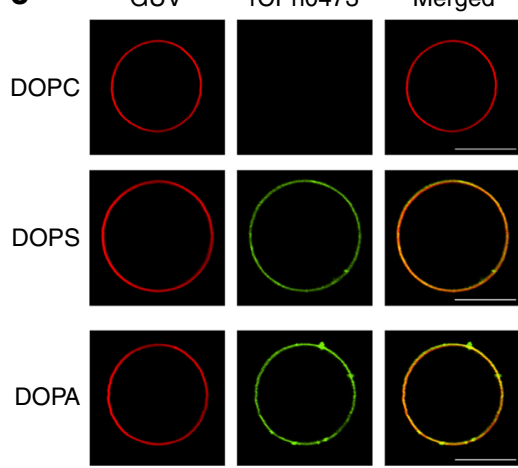

d
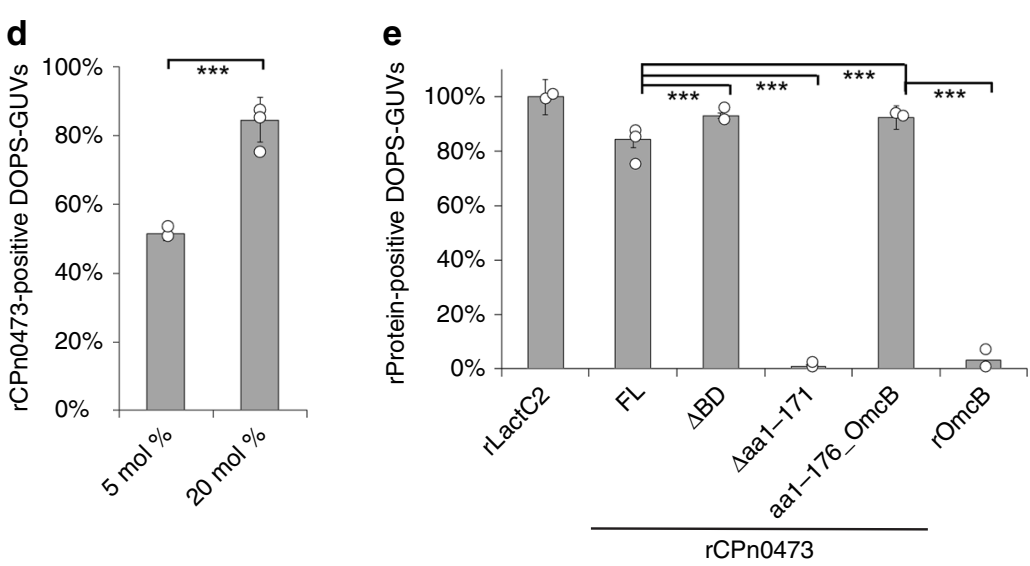

f

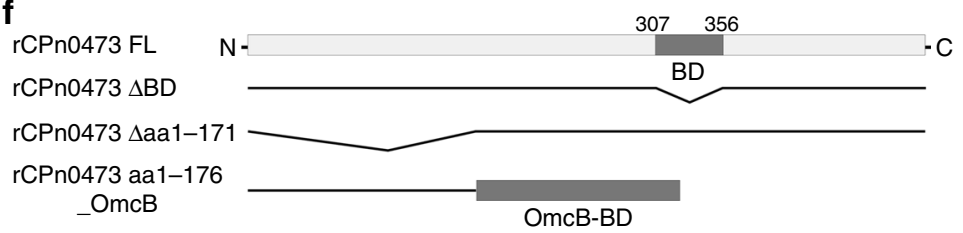

Fig. 1 Recombinant CPn0473 interacts preferentially with phosphatidylserine (PS). a Full-length rCPn0473 binds to liposomes ( $L-\alpha-$ phosphatidylcholine, phosphatidylethanolamine, carbohydrates and other lipids). Pellet (P) and supernatant (S) fractions were analyzed on Western blots ( $n=3)$. $\mathbf{b}, \mathbf{c}$ Binding efficiency of rCPno473 FL to giant unilamellar vesicles (GUVs). GUVs consisting of phosphatidylcholine (DOPC) plus the indicated lipids were incubated with DyLight488-labeled proteins (DOPS: phosphatidylserine, DOPA: phosphatidic acid, PI(4,5)P2: phosphatidylinositol 4,5-bisphosphate). ( $\geq 75$ GUVs were examined per experiment, $n=3$ ). Mean \pm standard deviation (s.d.). scale bar: $10 \mu \mathrm{m}$. d Effect of PS concentration on rCPnO473 binding. Mean \pm s.d. $(n=2)$. e Binding of rLactC2 ( $\geq 60$ GUVs examined per experiment, $n=3), r C P n 0473$ deletion variants ( $\geq 50$ GUVs examined per experiment, $n=2)$ and OmcB derivatives to GUVs. FL: full-length, BD: host-cell binding domain, aa: amino acid. Mean \pm s.d. $\mathbf{f}$ CPnO473 variants used in e. For all panels: ${ }^{\star \star \star} P<$ 0.001 , ${ }^{\star} P<0.01$ and ${ }^{\star} P<0.05$, n.s. not significant $(P>0.05)$ (Student's two-sample $t$ test). Source data are provided as a Source Data file

Importantly, exposure of HEp-2 cells to the chlamydial adhesins rPmp21 or rOmcB did not induce PS externalization (Supplementary Fig. 4a).

Next, we modulated the amount of CPn0473 on the surface of infectious EBs prior to infection of HEp-2 cells. Coating of EBs with $\mathrm{rCPn} 0473$ resulted in EB-associated PS signals that were more than three times as intense as those in untreated EBs, and correlated well with the total amount of CPn0473 associated with EBs (Fig. 3f, Supplementary Fig. 4b). Correspondingly, blocking endogenous CPn0473 accessible on the EB surface by pretreatment with anti-CPn0473 antibodies reduced PS externalization relative to untreated EBs (Fig. 3g, Supplementary Fig. 4b).
Exposure to an anti-Pmp21 antibody had little effect on PS externalization.

To identify the region of CPn0473 responsible for PS externalization, we first tested the variant lacking the $\mathrm{BD}$ domain (Supplementary Fig. 1d) ${ }^{10}$. rCPn0473 $\triangle B D$ is unable to bind HEp-2 cells ${ }^{10}$ and, as expected failed to induce PS externalization. Likewise, the deletion variant lacking the first 171 aa, which is incapable of PS-binding, was also unable to externalize PS. However, a variant lacking aa 150-255 was still able to externalize PS, indicating that the N-terminal aa 1-149 are essential for PS externalization (Supplementary Fig. 4c). 


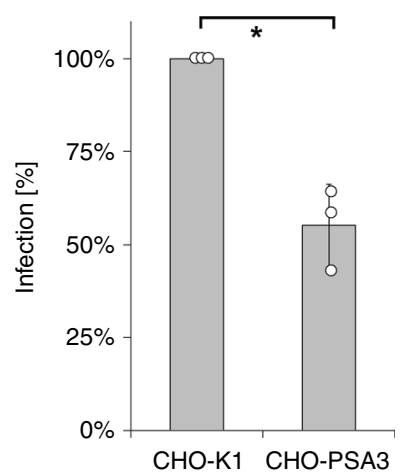

C
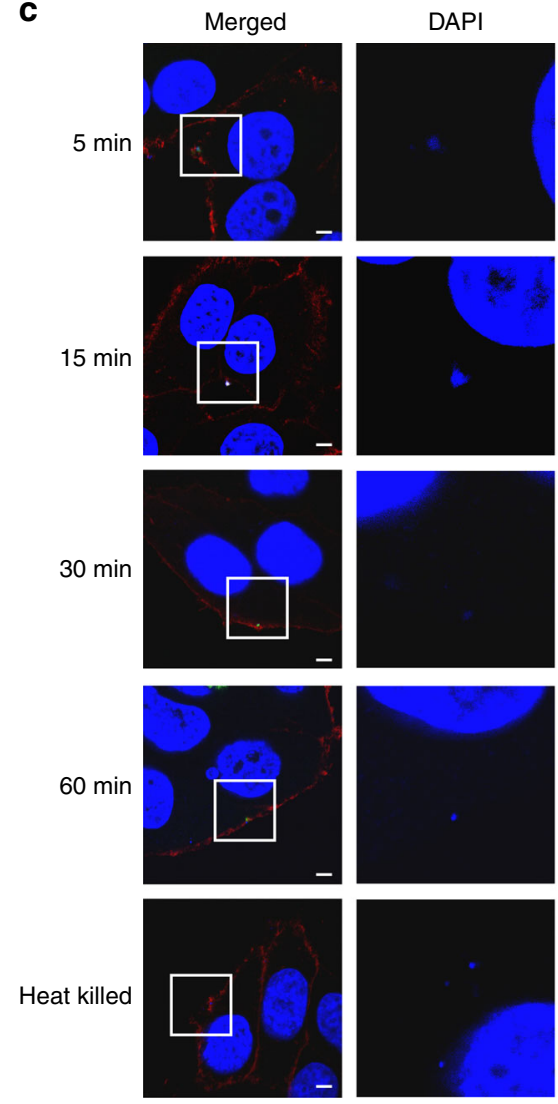

d

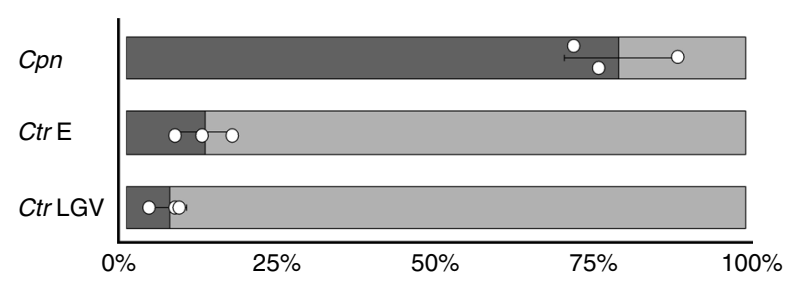

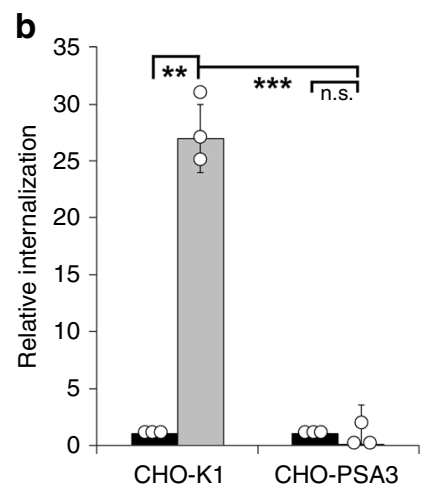

- BSA

$\square \mathrm{rCPn} 0473$
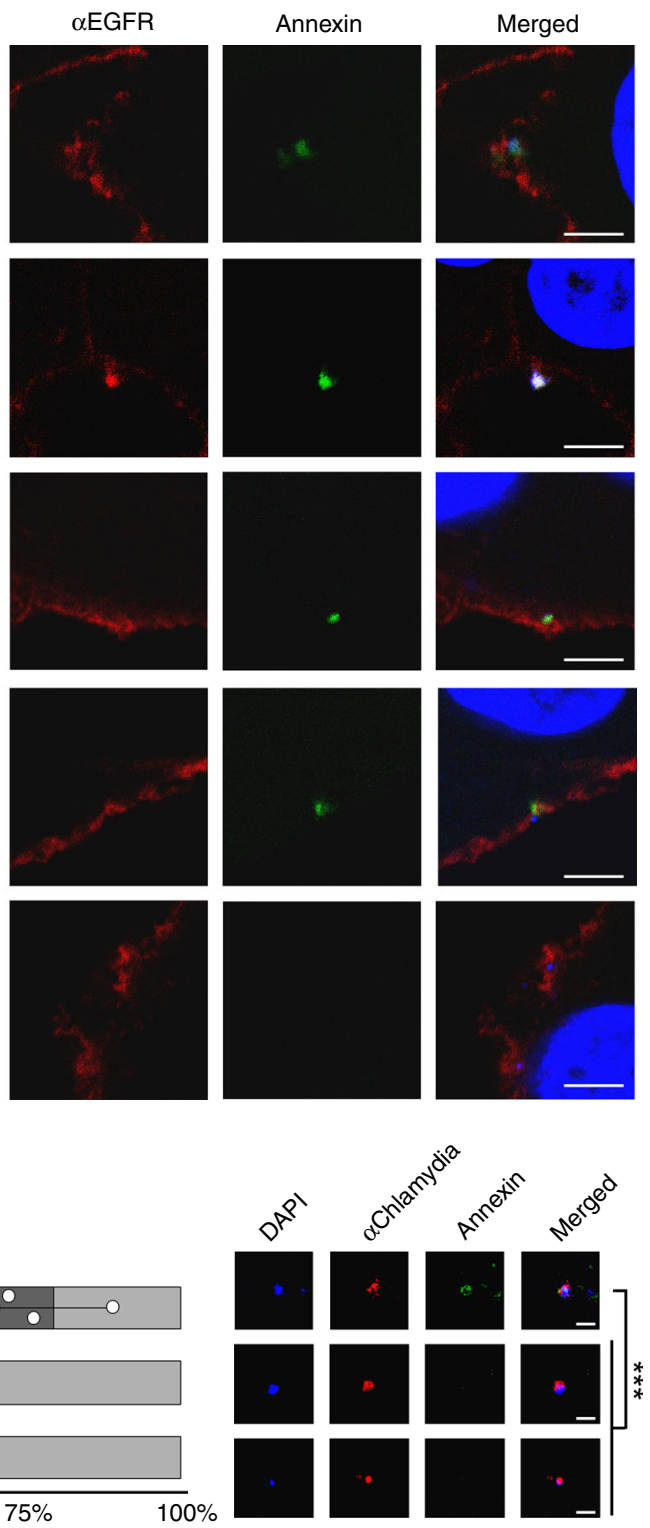

Fig. 2 C. pneumoniae specifically induces externalization of phosphatidylserine (PS). a Infectivity of C. pneumoniae EBs (MOI = 10) in wild-type (WT) and PS-deficient (PSA3) CHO cells $(n=3)$. mean \pm s.d. Infectivity in WT CHO cells was set to $100 \%$. Mean \pm s.d. $\mathbf{b}$ qRT PCR analysis of internalized Cpn. Triplicate samples of EBs in CHO WT and PSA3 cells ( $2 \mathrm{hpi})$ were pre-treated with $\mathrm{rCPnO} 473(100 \mu \mathrm{g} / \mathrm{ml})$ for $1 \mathrm{~h}$ prior to infection $(\mathrm{MOI}=10)(\mathrm{h}=3)$. Mean \pm s.d. c Externalization of PS by C. pneumoniae EBs early in infection. HEp-2 cells were infected with chlamydial EBs for the indicated times (MOI $=$ 10). Externalized PS was stained with annexin-V-FLUOS prior to fixation, followed by staining with DAPI and anti-EGFR antibody. Scale bars: $2.5 \mu$ m. d Externalization of PS by the indicated chlamydial species. HEp-2 cells were infected with chlamydial EBs for $1 \mathrm{~h}(\mathrm{MOI}=10)$. Externalized PS was stained as in c. Mean (triplicates) \pm s.d. $(n=3)$. For all panels: ${ }^{\star \star}{ }^{\star} P<0.001,{ }^{\star \star} P<0.01$ and ${ }^{\star} P<0.05$, n.s. not significant $(P>0.05)$ (Student's two-sample $t$ test). Source data are provided as a Source Data file 

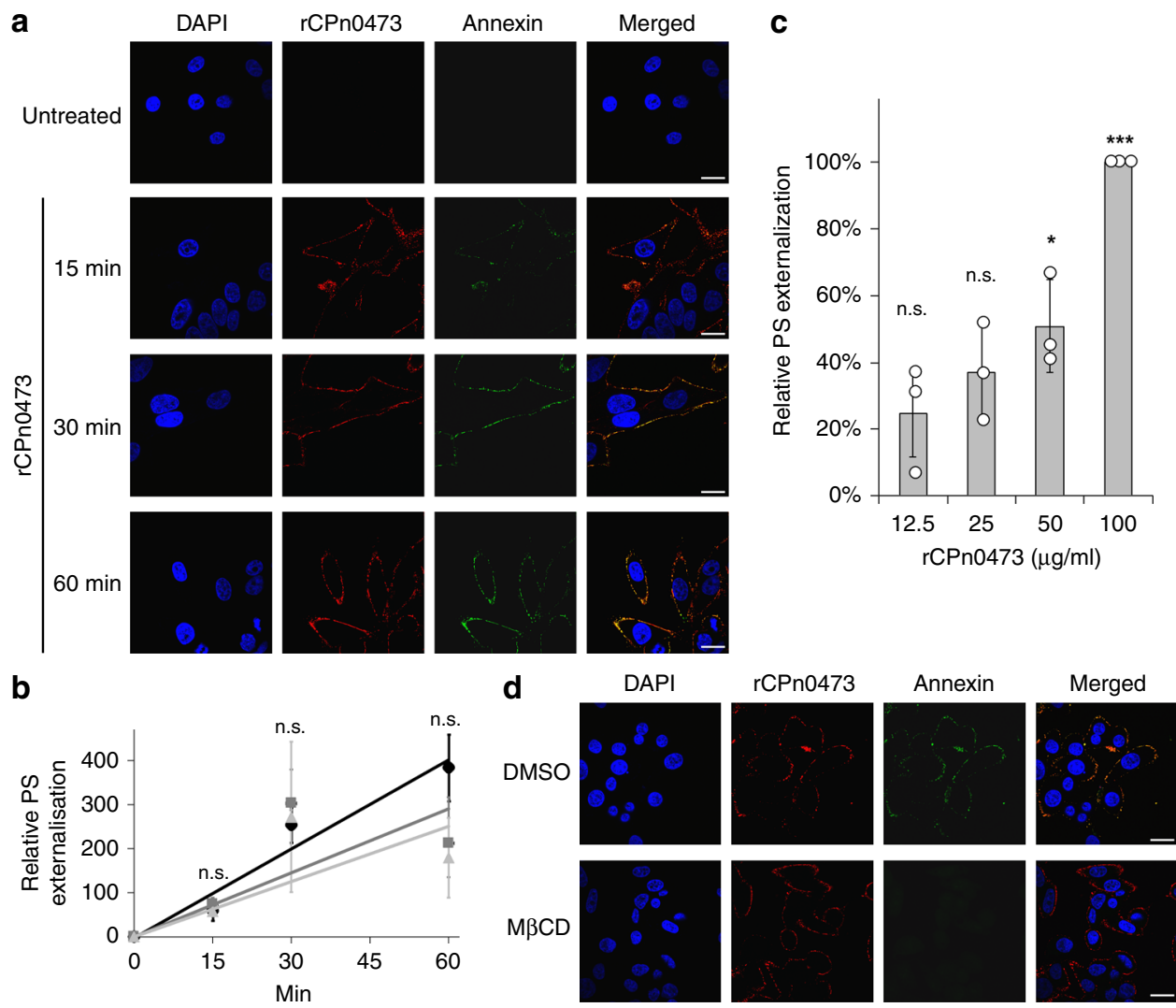

d

DAPI
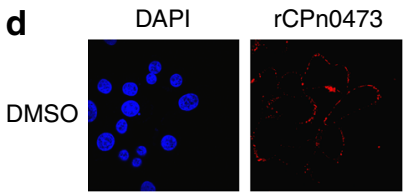

Annexin
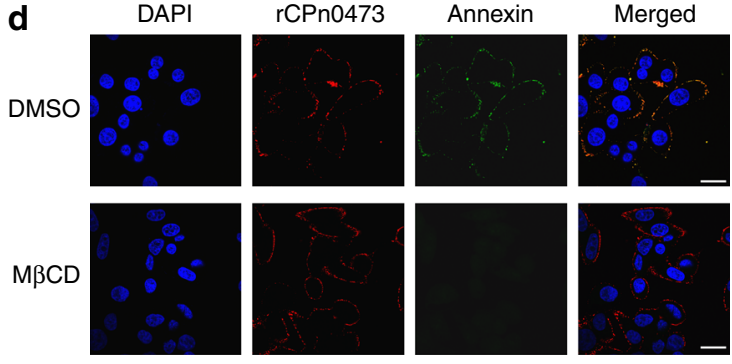

e

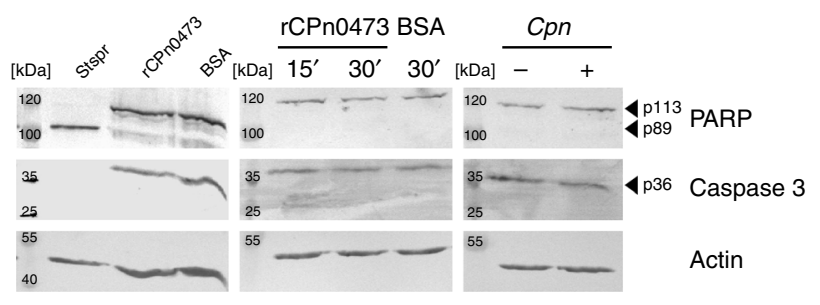

f

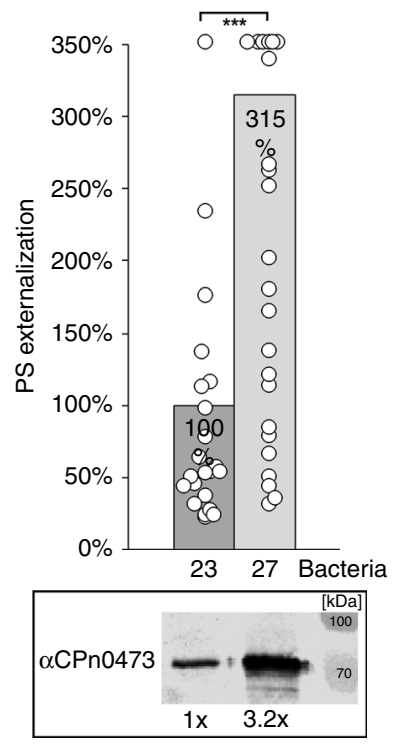

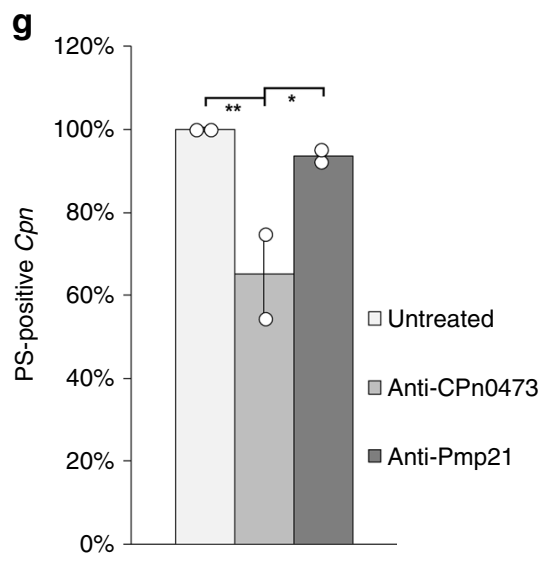

CPn0473 inserts into host membranes via its $C$ terminus. The data presented thus far are compatible with the hypothesis that $\mathrm{CPn} 0473$ itself is a PS translocator. To elucidate the membrane orientation of rCPn0473 during binding to the host-cell PM, Histagged rCPn0473 was site-specifically biotinylated at single cysteine (Cys) added to the $\mathrm{N}$ - or $\mathrm{C}$ terminus, and incubated with HEp-2 cells, which were then exposed to $\beta$-mercaptoethanol. If the biotinylated terminal Cys is accessible to reduction by $\beta$ mercaptoethanol, the biotin moiety should be lost. Indeed, after treatment of cells with the N-terminally biotinylated CPn0473, 
Fig. 3 Recombinant CPn0473 induces externalization of phosphatidylserine (PS). Externalized PS was stained using annexin prior fixation and antibody staining a-f. a-c Fluorescence analysis reveals time- and concentration-dependent PS externalization by rCPn0473. Representative images are shown in a, scale bars: $10 \mu \mathrm{m}$. b Fluorescence analysis of externalized PS on HEp-2 cells, incubated with recombinant CPn0473 (100 $\mu \mathrm{g} / \mathrm{ml})$ in DMEM media with different calcium levels (circle $(1.8 \mathrm{~mm})$, square $(2.8 \mathrm{~mm})$, triangle $(4.8 \mathrm{~mm})$ ). Mean \pm s.d. $(n=3)$. c Concentration dependence of PS externalization by rCPn0473. Mean \pm s.d. $(n=3)$. d Cholesterol depletion with $5 \mathrm{~mm} \mathrm{M \beta CD} \mathrm{reduces} \mathrm{the} \mathrm{efficiency} \mathrm{of} r C P n 0473$-induced PS externalization. Scale bars: $10 \mu \mathrm{m}$. e Western blot analysis of apoptosis marker proteins (caspase-3 and PARP). Apoptosis was induced by incubation with $2.5 \mu \mathrm{M}$ staurosporine (Stspr). Arrowheads mark the inactive procaspase-3 (p36), full-length PARP (p113), and cleaved PARP (p89) $(n=3)$. f Coating of C. pneumoniae EBs with $1 \mu \mathrm{M}$ rCPn0473 at $1 \mathrm{hpi}(\mathrm{MOI}=10)$ boosts levels of PS externalization. CPn0473 levels were determined by Western blotting. The mean of one typical

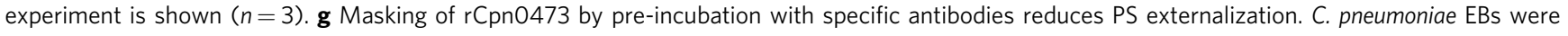
pre-incubated with $\mathrm{CPn0473-specific} \mathrm{antibodies} \mathrm{(diluted} \mathrm{1:100)} \mathrm{at} \mathrm{MOI}=20$. At least $70 \mathrm{DAPI}$ - and CPn0473-positive EBs analyzed per experiment. Mean \pm s.d. $(n=2)$. For all panels: ${ }^{\star \star \star} P<0.001,{ }^{\star \star} P<0.01$, and ${ }^{\star} P<0.05$, n.s. not significant $(P>0.05)$ (Student's two-sample $t$ test). Source data are provided as a Source Data file

only $6 \pm 5 \%$ of the protein remained biotinylated, whereas $39 \%$ $( \pm 1 \%)$ of the C-terminally modified form survived exposure to the reductant, indicating that the $\mathrm{C}$ terminus may be inserted into the host PM (Fig. 4a). Indeed, a transmembrane region is predicted near the C terminus (TM, aa 396-425) by ProtScale Hphob $^{18}$ (Fig. 4b). To determine whether rCPn0473 binding alters membrane integrity, we monitored the stability of carboxyfluorescein (CF)-loaded DOPS-GUVs in the presence of Alexa594-labeled full-length (FL) rCPn0473 or a TM deletion variant $(\triangle \mathrm{TM})$. Upon incubation with $\mathrm{rCPn} 0473 \mathrm{FL}$, the $\mathrm{CF}$ signal within the GUV lumen decreased within $5 \mathrm{~min}$, and this was followed by an increase in the luminal Alexa594 signal. Although a $50 \%$ loss of CF (MW: $376.32 \mathrm{Da}$ ) was observed within $\sim 2.5 \mathrm{~min}$, it took $8.5 \mathrm{~min}$ to reach $50 \%$ of the maximal Alexa594rCPn0473 (MW: $55 \mathrm{kDa}$ ) uptake into the GUV lumen. In contrast, binding of the $\Delta \mathrm{TM}$ variant to PS-containing GUVs did not alter luminal $\mathrm{CF}$ fluorescence nor result in increased luminal Alexa594 fluorescence (Fig. 4c-e). Thus, rCPn0473 binding to DOPS-GUVs makes the membrane permeable to small and large molecules without affecting overall GUV stability.

CPn0473 is a chlamydial PS translocator. In order to test directly for PS translocator activity of rCPn0473, we prepared GUVs bearing PS predominantly in one of the membrane leaflets ${ }^{19,20}$. PS asymmetry was verified using FITC-rLactC2. Mixing GUVs carrying PS in the outer leaflet ( $\left.\mathrm{PS}_{\text {out }}-\mathrm{GUV}\right)$ with rLactC2 immediately resulted in strong FITC fluorescence at time point $0 \mathrm{~min}$, which declined over time. GUVs carrying PS localized mainly in the inner leaflet $\left(\mathrm{PS}_{\mathrm{inn}}\right.$-GUV) showed little FITCrLactC2 fluorescence under the same conditions (Fig. 4f, g). However, when $\mathrm{PS}_{\text {inn }}$-GUVs were preloaded with FITC-rLactC2, the luminal rLactC2 stained inner leaflet PS, resulting in significant fluorescence and confirming PS asymmetry (Fig. 4f, Supplementary Fig. 4d, e).

Crucially, simultaneous exposure of $\mathrm{PS}_{\text {inn }}$-GUVs to external rCPn0473 and FITC-rLactC2 immediately resulted in FITCrLactC2 fluorescence, which increased over the next $30 \mathrm{~min}$ (Fig. 4f, g). On the other hand, when PS $_{\text {out }}$-GUVs, prepacked with FITC-rLactC2, were exposed to external rCPn0473, we observed no FITC-rLact2 fluorescence at the inner leaflet (Supplementary Fig. $4 \mathrm{~d}, \mathrm{e})$. These results argue against a scrambling mechanism for rCPn0473, and thus support directional rCPn0473-mediated PS translocation from the inner (PS-high) to the outer (PS-low) leaflet in a synthetic membrane system.

\section{Discussion}

Our data identify the Cpn adhesin CPn0473 as a lipid translocator that mediates PS externalization at the host-cell PM surrounding the adhering pathogen. We therefore refer to the protein hereafter as LIPP (for 'Lipid-dependent Internalization Promoting
Protein'). LIPP is found on the surface of adhering Cpn $\mathrm{EBs}^{10}$. Our model implies that LIPP interacts via its BD domain with the $\mathrm{PM}$ via an as yet unknown cell surface structure associated with cholesterol-rich microdomains. The LIPP-PM interaction likely triggers both conformational changes within the protein and oligomerization. Together, these changes enable the N-terminal domain of LIPP to interact with the PM and translocate PS from the inner to the outer leaflet of the bilayer-which in turn enhances EB internalization. This scenario could explain why the assembly and the activity of the LIPP translocation complex acts rather slowly by comparison with known integral membrane flippases and scramblases. Notably, LIPP activity requires neither ATP nor $\mathrm{Ca}^{2+}$, although a potentially beneficial effect of intracellular ATP- or $\mathrm{Ca}^{2+}$-stores cannot be ruled out at the moment. LIPP is therefore the first known lipid translocator protein that can gain access to the inner leaflet of the PM from the extracellular side.

How might exofacial PS localization support EB internalization? CPn is an obligate intracellular pathogen, and EB internalization critical for infection. Thus $C p n$ may have invented PL translocase activity as a mechanism to expose a specific host-cell ligand, i.e., PS, to which the LIPP adhesin can then bind. Surfaceexposed PS also acts as a ligand during apoptotic clearance and blood cell clotting. Moreover, PS externalization promotes HIV uptake $^{21}$. However, in these latter cases, PS exposure occurs via activated human scramblases. Exofacial PS has also been found to have a role during host-cell entry by other bacterial pathogens, but the underlying mechanisms are not understood ${ }^{21-23}$.

Translocation of PS will also modulate PM structure. Under homeostatic conditions, the anionic PS is restricted to the inner leaflet of the PM and its head-group experiences electrostatic repulsion, which is limited by cholesterol (also enriched in the inner leaflet ${ }^{24,25}$. Specific PS translocation around the adhering EB increases the anionic charge density of the exofacial leaflet which, in the absence of sufficient cholesterol, will develop outward curvature at the edges of the invaginating membrane owing to electrostatic repulsion, thus supporting EB endocytosis. This process might be facilitated by host scaffolding proteins, which are known to enhance membrane curvature and thereby vesicle formation $^{26}$. Finally, transient PS depletion from the inner PM leaflet surrounding the adhering Cpn EBs will result in a relocalization of specific PS-binding proteins normally found associated with the inner bilayer leaflet, which would otherwise impinge on the entry process. Hence, our findings not only contribute to our knowledge of basic host-pathogen interactions, they suggest that inhibiting PS exposure and/or pathogen-PS interaction might open a route to therapies for bacterial and viral diseases.

\section{Methods}

Chemicals and antibodies. $\mathrm{M} \beta \mathrm{CD}$, nocodazole, Cell Dissociation Solution Nonenzymatic $1 \times$, AP-coupled streptavidin, CF and FITC-conjugated cholera toxin B 
a

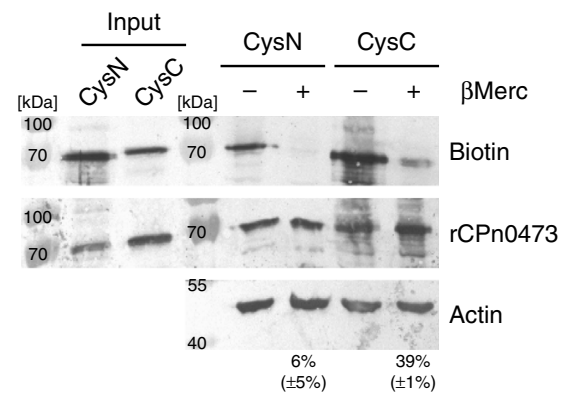

C

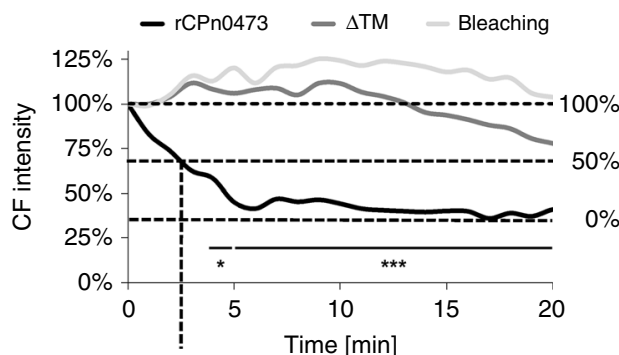

d

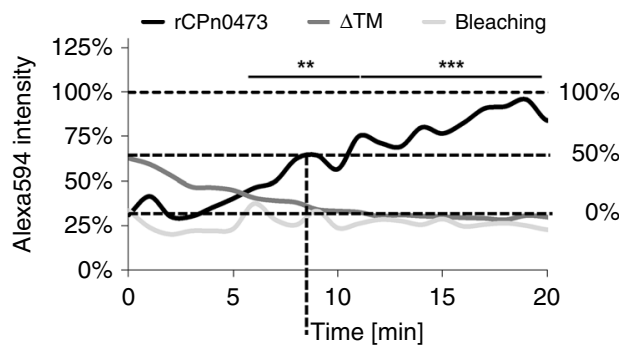

$\mathbf{f}$
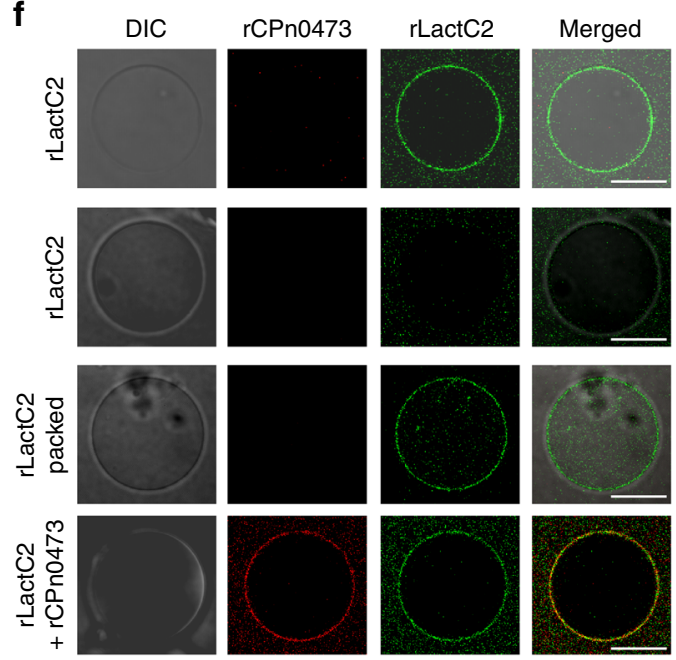

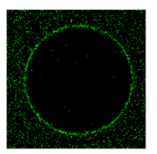

b

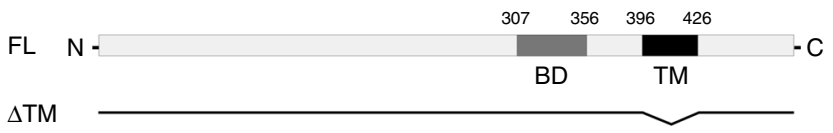

e
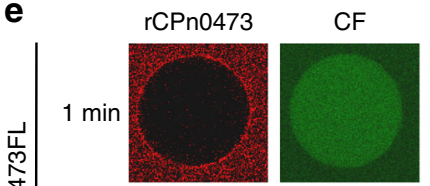

Merged

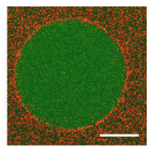

$20 \min$
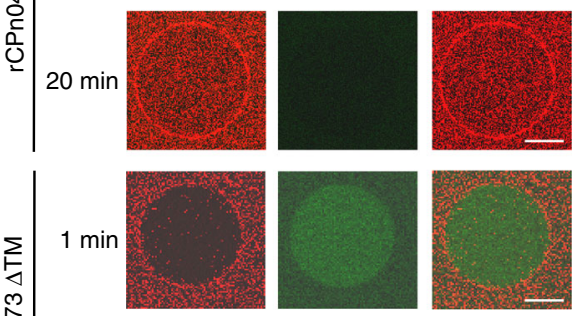

$20 \min$
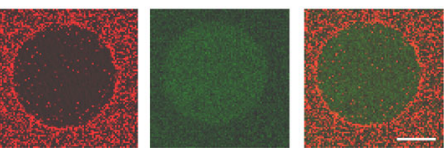
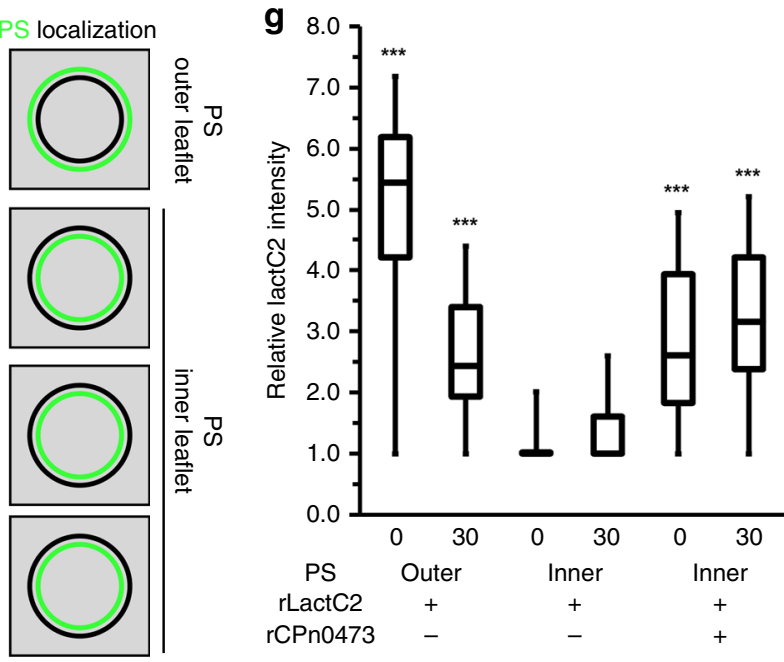

subunit (FITC-CTxB) were obtained from Sigma-Aldrich,

carboxyfluoresceindiacetate-succinimidylester (CFSE) and the lipids DOPS, DOPC, PI(3)P, PI(4,5) $\mathrm{P}_{2}$, DOPA, TR-DHPE from Life Technologies, gentamicin was sourced from Invitrogen, proteinase K from Millipore and the SYBR Green PCR Master Mix from Applied Biosystems. Staurosporine, anti-caspase-3 antibodies $(1: 100$, Western blot (WB)), anti-PARP antibodies $(1: 100, W B)$, and annexin- $V$ were kindly provided by Christoph Peter (Institute for Molecular Medicine, HHU, Düsseldorf). Annexin was also obtained directly from Roche. EZ-Link HPDPbiotin, cytochalasin $\mathrm{D}$ and rhodamine/phalloidin was obtained from Thermo Scientific, the lipid membrane strips were from Echelon Biosciences. Commercially available antibodies were obtained from the following sources: anti- $\beta$-actin (\#A1978, 1:2000 (WB)) from Sigma-Aldrich; Alexa488- and Alexa594-conjugated anti-rabbit and anti-mouse antibodies from Life Technologies (all 1:200
(Immunofluorescence (IF)); anti-GST (\#C2309, 1:1000 (WB)) from Santa Cruz; anti-PentaHis (\#34660, 1:2500 (WB)) from Qiagen; AP-conjugated anti-mouse $(\#$ S372, 1:7500 (WB)) and anti-rabbit (\#S3731, 1:7500 (WB)) from Promega; anti-LPS (Pathfinder) (\#30701, 1:4 (IF)) from BioRad, anti-tubulin from Acris, anti-EGFR (\# MA5-13269, 1:50 (EGFR)) from Invitrogen. The anti-Pmp21 (1:50 (WB); 1:10 (IF)) and anti-CPn0473 (1:25 (WB); 1:5 (IF)) antibodies were produced in rabbit by Eurogentec, and anti-MOMP (1:100 (WB)) was generated by G. Zhong (University of Texas Health Science Center at San Antonio). Phosphate-buffered saline (PBS) buffer used in the experiments consists of $137 \mathrm{~mm}$ $\mathrm{NaCl}, 2.7 \mathrm{~mm} \mathrm{KCl}, 10 \mathrm{~mm} \mathrm{Na} \mathrm{HPO}_{4}, 1,8 \mathrm{~mm} \mathrm{KH}_{2} \mathrm{PO}_{4}$. Hank's Buffered Saline Solution (HBSS) (Thermo Scientific, \#14175-053) used in this work consists of $5.3 \mathrm{~mm} \mathrm{KCl}, 0.4 \mathrm{~mm} \mathrm{KH} \mathrm{PO}_{4}, 4.2 \mathrm{~mm} \mathrm{NaHCO} 3,137.9 \mathrm{~mm} \mathrm{NaCl}, 0.3 \mathrm{~mm} \mathrm{Na} \mathrm{HPO}_{4}$, $5.6 \mathrm{~mm}$ D-Glucose. 
Fig. 4 rCPn0473 acts as a chlamydial phosphatidylserine translocase. a Orientation of $\mathrm{rCPnO} 473$ in the host-cell membrane. N- or C-terminal singlecysteine variants of $\mathrm{CpnO473}$ were biotinylated prior to incubation with $\mathrm{HEp}-2$ cells. Accessible (i.e., extracellular) biotin attached to the terminal Cys residue was removed by treatment with $\beta$-mercaptoethanol and the amount that remained linked to the protein was analyzed on Western blots using streptavidin. Mean residual biotin is given underneath the Western blot $(n=3)$. b Schematic of a CPn0473 deletion variant lacking the predicted transmembrane domain ( $\triangle T M$ ) predicted by ProtScale Hphob. BD: host-cell binding domain. c-e Binding of rCpn0473 affects the integrity of PS-containing GUVs. DOPS-GUVs were preloaded with carboxyfluorescein (CF, 1:100 dilution) and incubated with Alexa594-coupled rCPn0473, rCPn0473 $\Delta T M$ or untreated (Bleaching). Fluorescence intensity of CF $\mathbf{c}$ and Alexa594 $\mathbf{d}$ in the GUV lumen was analyzed (at least 15 GUVs) ( $n=2$ ). The horizontal dotted lines show the differences in signal intensity (0\%,50\% and 100\% signal intensity) during the course of the experiment. The dotted vertical line represents the time point when a $50 \%$ shift in signal intensity is reached. Representative images are shown in e. Scale bar: $10 \mu \mathrm{m}$. Significant differences between fulllength rCPn0473 and rCPn0473 4 TM are indicated. f, g Asymmetric GUVs were prepared based on ${ }^{19}$. In the presence or absence of rCPn0473, FITClabeled, recombinant rLactC2, as a marker for PS, was incubated with GUVs bearing PS predominantly in the outer (PS out $-G U V)$ or inner (PS inn-GUV) leaflet. Representative views are shown in $\mathbf{f}$. Scale bar: $10 \mu \mathrm{m}$. rLactC2 binding was quantified using ImageJ software. Background intensity was set to 1 (up to 24 GUVs analyzed) $(n=3)$. g. The data are represented as a box and whisker plot. The center line represents the mean score, the upper and lower quartiles contain the mean $50 \%$ of the data while the whiskers show the minima and maxima of all data. Significance of differences is determined between individual time points ( $0 \mathrm{~min}$ or $30 \mathrm{~min}$ ). For all panels: ${ }^{\star \star \star} P<0.001,{ }^{\star \star} P<0.01$, and ${ }^{\star} P<0.05$ (Student's two-sample $t$ test). Source data are provided as a Source Data file

\begin{abstract}
DNA manipulations and protein expression. Escherichia coli $\mathrm{XL}_{1}$ blue (Stratagene) was used for plasmid amplification and the Origami strain (Novagen) for protein expression. Plasmids were constructed by in vivo homologous recombination in Saccharomyces cerevisiae strain YKM2, a GFP-expressing version of EBY100 (Invitrogen). All coding sequences were amplified via PCR from chlamydial genomic DNA or cDNA derived from human genomic DNA with a 40 base pair long homologous region on either side for cloning into the respective destination vector in S. cerevisiae ${ }^{6}$ (Supplementary Table 1). The binding domain of OmcB (aa 40-100) from C. pneumoniae was expressed with a C-terminal His-tag and fused N-terminally to GST. GST, Pmp21, CPn0473 FL and the deletion variants were expressed as C-terminally His-tagged proteins. The PS-binding domain of lactadherin (expression clone kindly provided by Dr. S. Grinstein, University of Toronto, Toronto, Canada ${ }^{16}$ (Addgene Plasmid \# 15247)) was C terminally tagged with GST. The PI $(4,5) \mathrm{P}_{2}$-binding domain of phosphoinositide-phospholipase $\mathrm{C}$ gamma (PLC $\gamma$ ) and the PI(3)P-binding FYVE domain of the Hepatocyte growth factor-regulated tyrosine kinase substrate $(\mathrm{Hgs} / \mathrm{Hrs})$ were $\mathrm{C}$-terminally tagged with GST (kindly provided by Dominik Spona, HHU, Düsseldorf). GST- and His-tagged proteins were purified using the protocols supplied by Sigma-Aldrich and Qiagen, respectively, and analyzed on Western blots.
\end{abstract}

Cultivation of epithelial cells. Human HEp-2 cells (epithelial larynx carcinoma, ATCC No.: CCL-23) were cultivated in Dulbecco's Modified Eagle Medium (DMEM) GlutaMax with fetal calf serum (FCS), vitamins, non-essential amino acids, amphotericin B $(2.5 \mu \mathrm{g} / \mathrm{ml})$ and gentamicin $(50 \mu \mathrm{g} / \mathrm{ml})$ at $37^{\circ} \mathrm{C}$ in an atmosphere containing $6 \% \mathrm{CO}_{2}$. $\mathrm{CHO}$ cells (Chinese hamster ovary, ATCC No.: CCL. 61) were cultivated in F12K medium supplemented with FCS, vitamins, nonessential amino acids, amphotericin B $(2.5 \mu \mathrm{g} / \mathrm{ml})$ and gentamicin $(50 \mu \mathrm{g} / \mathrm{ml})$ at 37 ${ }^{\circ} \mathrm{C}$ in an atmosphere containing $6 \% \mathrm{CO}_{2}$. Wild-type $\mathrm{CHO}-\mathrm{K} 1$ and the PS-deficient $\mathrm{CHO}$ cell line PSA3 (defective in the pssA gene encoding PS synthase I activity) were kindly provided by Dr. O. Kuge, Kyushu University, Fukuoka, Japan ${ }^{13}$. The CHO-PSA3 was cultivated using F12K medium containing PS liposomes $(30 \mu \mathrm{M})$.

Propagation of chlamydial strains. The HEp-2 cell line was used for propagation of chlamydial strains (patient isolates). The cells were cultured in DMEM GlutaMax with FCS, vitamins, non-essential amino acids, amphotericin B $(2.5 \mu \mathrm{g} / \mathrm{ml})$ and gentamicin $(50 \mu \mathrm{g} / \mathrm{ml})$ at $37^{\circ} \mathrm{C}$ in an atmosphere containing $6 \% \mathrm{CO}_{2} . \mathrm{C}$. pneumoniae $\mathrm{GiD}$ and the $C$. trachomatis serovars L2 (L2/434/Bu) and E (DK-20) were propagated in HEp-2 cells in the presence of $1.2 \mu \mathrm{g} / \mathrm{ml}$ cycloheximide, and EBs were purified using $30 \%$ gastrografin (Schering). The $\mathrm{CHO}$ cell lines were used for adhesion and infection experiments.

Immunofluorescence microscopy. Microscopy was performed with a Nikon Eclipse Ti-E C2 confocal microscope (DS-QilMC Camera). Confocal images were assembled using NIS Element software (Nikon). Infected cells were fixed either with $96 \%$ methanol for $5 \mathrm{~min}$ at room temperature or with $3 \%$ para-formaldehyde for $20 \mathrm{~min}$ at $4{ }^{\circ} \mathrm{C}$. Where necessary, cells were permeabilized with $2 \%$ saponin prior to staining with an antibody or marker protein. In this case, $0.5 \%$ saponin was present during the whole staining process. Unspecific binding sites were blocked with $2 \% \mathrm{BSA}$ and antibodies were dissolved in a $0.05 \%$ Tween-20 solution.

Adhesion assay. Adhesion assays were carried out with fully confluent layers of $10^{6} \mathrm{HEp}-2$ cells or $\mathrm{CHO}$ cells grown at $37^{\circ} \mathrm{C}$. Binding of soluble recombinant proteins was assessed by overlaying confluent cells with $250 \mu$ l of culture medium containing the soluble recombinant protein of interest $(100 \mu \mathrm{g} / \mathrm{ml})$ at $4{ }^{\circ} \mathrm{C}$ on ice for 15, 30, or $60 \mathrm{~min}$. After extensive washing, cells were detached, pelleted ( $5 \mathrm{~min}$ at $300 \times g)$ and resuspended in HBSS $(100 \mu \mathrm{l})$. Bound recombinant protein was then quantified by immunoblotting using anti-His antibodies.

Membrane localization assay. To assay the orientation of $\mathrm{rCPn} 0473 \mathrm{FL}$ when bound to the PM of host cells, the C-terminally His-tagged protein was modified by adding a single cysteine (Cys) at either the $\mathrm{N}$ - or the $\mathrm{C}$ terminus. The two variants were then biotinylated at the terminal Cys residue in the presence of 1 mM EDTA using $0.05 \mu \mathrm{g}$ of EZ-Link HPDP-Biotin per $\mu \mathrm{g}$ protein for $4 \mathrm{~h}$ on ice. Each of the two biotinylated forms $(100 \mu \mathrm{g} / \mathrm{ml})$ were incubated with confluent HEp-2 cells at $37^{\circ} \mathrm{C}$ for $30 \mathrm{~min}$. Cells were cooled on ice and incubated with $100 \mathrm{~mm} \beta$ mercaptoethanol $(10 \mathrm{~min})$ to remove extracellular biotin. After extensive washing, cells were detached, using Cell Dissociation Solution, pelleted ( 5 min at $300 \times g$ ) and resuspended in HBSS $(100 \mu \mathrm{l})$. Bound recombinant protein was then quantified by immunoblotting using anti-His antibodies. The amount of terminally Cysbound biotin remaining was measured using AP-coupled streptavidin and quantified relative to the anti-His signal and the loading control using ImageJ.

Infection assays using a PS-deficient cell line. Wild-type CHO-K1 and the PSdeficient $\mathrm{CHO}$ cell line PSA $3^{13}$ used in infection assays were starved for 2 days in F12K medium lacking PS liposomes $(30 \mu \mathrm{M})$. Prior to infection with EBs $(\mathrm{MOI}=$ 40 ) at $2 \mathrm{hpi}$, the medium was replaced by F12K medium containing PS liposomes $(30 \mu \mathrm{M})$, prepared via the freeze-thaw-sonification method (explained below), and cycloheximide $(1.2 \mu \mathrm{g} / \mathrm{ml})$. At $50 \mathrm{hpi}$, cells were washed with HBSS and fixed with methanol. Inclusions were stained with Pathfinder. Infectivity was quantified by the standard microscopy assay by counting the numbers of inclusions in three large images (size of $3 \times 3$ single pictures, 60 -fold magnification) (triplicates).

To quantify the effects of recombinant proteins on $\mathrm{EB}$ internalization, confluent $\mathrm{CHO}$ cells were overlaid with $250 \mu \mathrm{l}$ of cell culture medium containing the relevant recombinant protein $(100 \mu \mathrm{g} / \mathrm{ml})$, incubated for $1 \mathrm{~h}$, and cooled to $4^{\circ} \mathrm{C}$. Purified EBs were added (at MOI $=40$ ) and incubated for $1 \mathrm{~h}$ at $4^{\circ} \mathrm{C}$, then for $2 \mathrm{~h}$ at $37^{\circ} \mathrm{C}$ to allow internalization. After removal of unbound EBs by extensive washing with HBSS, CHO cells and non-internalized EBs were detached with $0.5 \times$ Trypsin/ EDTA solution (Invitrogen) for $15 \mathrm{~min}$ at $37^{\circ} \mathrm{C}$. EBs were removed by sequential centrifugation at $1000 \times g$ and $250 \times g$. Internalization was quantified by qPCR using a modified version of a previously described protocol ${ }^{27}$. DNA was isolated from infected cells by phenol-chloroform extraction and QPCR was performed with 200 ng of RNase-treated DNA using the SYBR Green PCR Master Mix (Applied Biosystems) and $16 \mathrm{~S}$ rRNA primers (forward: CCAACACCTCACGGCACGAG, reverse: CGCCTGAGGAGTACACTCGC) to detect chlamydial DNA and GAPDH primers (forward: TGGCTACAGCAACAGAGTGG, reverse:

GTGAGGGAGATGATCGGTGT) to quantify host DNA. The reaction sequence for qPCR consisted of $5 \mathrm{~min}$ at $95^{\circ} \mathrm{C}$, followed by 60 cycles of $95^{\circ} \mathrm{C}$ for $15 \mathrm{~s}, 60^{\circ} \mathrm{C}$ for $15 \mathrm{~s}$ and $68^{\circ} \mathrm{C}$ for $30 \mathrm{~s}$ (ABI Prism 7000, Applied Biosystems). The number of $16 \mathrm{~S}$ gene copies relative to GAPDH was expressed as $2^{-\Delta \Delta C T 27}$.

Membrane lipid strip assay. PL-binding assays were carried out using nitrocellulose membrane strips bearing (100 pmol) samples of 15 different biologically active lipids (Echelon Biosciences Inc.), following the protocol provided by the supplier. The lipids tested were glyceryl tripalmitate (GT), diacylglycerol (DAG), PA, PS, phosphatidylethanolamine (PE), phosphatidylcholine (PC), phosphatidylglycerol (PG), cardiolipin (CL), phosphatidylinositol (PI), phosphatidylinositol(4)-monophosphate, $(\mathrm{PI}(4) \mathrm{P}$, phosphatidylinositol- $(4,5)$-diphosphate (PI(4,5)P2), phosphatidylinositol- $(3,4,5)$-triphosphate $(\mathrm{PI}(3,4,5) \mathrm{P} 3)$, cholesterol $(\mathrm{Chol})$, sphingomyelin (SM), and 3-sulphogalactosyl ceramide (Sulfatide). The nitrocellulose membranes were blocked with PBS-T $(0.1 \% \mathrm{v} / \mathrm{v}$ Tween) containing $3 \%(\mathrm{wt} / \mathrm{vol})$ fatty acid-free BSA for $1 \mathrm{~h}$ at room temperature (RT), following $1 \mathrm{~h}$ of incubation with the recombinant proteins $(1 \mu \mathrm{g} / \mathrm{ml})$ in PBS-T $(+3 \% \mathrm{BSA})$. The membranes 
were then washed three times with PBS-T, and bound recombinant protein was detected using anti-His or anti-GST antibodies. as described above. Only 4',6-diamidino-2-phenylindole-stained and anti-LPS (Cpn) or anti-MOMP (C. trachomatis) positive chlamydial EBs were counted.
Liposome pull-down experiment. Liposomes made of 55\% L-a-DOPC, 20\% phosphatidylethanolamine, carbohydrates and other lipids (Sigma-Aldrich, Type IV-S, \#P-3644) were prepared via the freeze-thaw-sonification method (generous gift from J. Wiese, Heinrich-Heine University Düsseldorf). Lipids were resuspended in chloroform and acetone and stirred for $2 \mathrm{~h}$ at RT. After precipitation of the PLs overnight at $4{ }^{\circ} \mathrm{C}$, lipids were dissolved in diethyl ether and dried under nitrogen. The PL-pellets were dissolved in HBSS and sonicated in a water bath $(30 \times 5 \mathrm{~s})$. They were purified by passage over a PD10 column and incubated with recombinant protein $(5 \mu \mathrm{g})$ for $20 \mathrm{~min}$ at room temperature in a final volume of $100 \mu \mathrm{l}$. Liposomes were pelleted for $15 \mathrm{~min}$ at $16000 \times g$, the supernatant was collected and the pellet was resuspended in $100 \mu \mathrm{l}$ PBS. Aliquots of the pellet and supernatant fractions were then analyzed by immunoblotting. Recombinant proteins were detected using anti-His antibodies.

GUV-binding assay. GUVs were prepared via electroformation as essentially described ${ }^{28,29}$. Lipid-coated ITO slides were dried under vacuum overnight. The following lipid mixtures (in mol\%) were spread on two ITO slides [the fluorescent lipid Texas Red-DHPE (Life Technologies) was added for imaging purposes]: DOPC/Texas Red-DHPE: 99.75/0.25, DOPC/TR-DHPE/cholesterol: 74.75/0.25/25, 94.75/0.25/5 DOPC/TR-DHPE/DOPS, 74.75/0.25/5/20 DOPC/TR-DHPE/DOPS/ cholesterol, 59.75/0.25/20/20, DOPC/TR-DHPE/DOPS/cholesterol, 94.75/0.25/5 DOPC/TR-DHPE/DOPA, 74.75/0.25/5/20 DOPC/TR-DHPE/DOPA/cholesterol, DOPC/TR-DHPE/DOPS/cholesterol, 94.75/0.25/5 DOPC/TR-DHPE/PI(4,5) $\mathrm{P}_{2}$, 74.75/0.25/5/20 DOPC/TR-DHPE/PI(4,5) $\mathrm{P}_{2} /$ cholesterol, 69.75/0.25/10/20 DOPC/ TR-DHPE/PI(4,5) $\mathrm{P}_{2} /$ cholesterol or $69.75 / 0.25 / 10 / 20$ DOPC/TR-DHPE/PI(3)P/ cholesterol. The two ITO slides were combined with the space between filled with sucrose $(10 \%, \mathrm{w} / \mathrm{v})$ and incubated at RT for $1.5 \mathrm{~h}$ (electroformation). The GUVs were added at room temperature to DyLight488-labeled rCPn0473 $(100 \mu \mathrm{g} / \mathrm{ml})$ and immediately observed on a confocal fluorescence microscope (Nikon Eclipse Ti-E with A1R confocal laser scanner, $60 \mathrm{x}$ oil objective, NA $=1.49)$. Image acquisition and analysis was performed with NIS-Elements (Nikon).

GUV lipid translocator assay. Asymmetric GUVs were prepared as essentially described previously ${ }^{19}$. The following lipid mixtures (in mol\%) were used for the preparation of GUVs: 60/20/20, DOPC/DOPS/cholesterol and 80/20, DOPC/cholesterol. A total of $115 \mu \mathrm{g}$ lipid mass per membrane leaflet was dried and resuspended in mineral oil $(200 \mu \mathrm{l}$ per leaflet). The outer leaflet was prepared by overlaying $500 \mu \mathrm{l}$ glucose $(1 \mathrm{M})$ with $200 \mu \mathrm{l}$ of the lipid mixture. The inner leafle was prepared by overlaying $50 \mu \mathrm{l}$ sucrose $(1 \mathrm{M})$ with $200 \mu \mathrm{l}$ of lipid mixture and mixed by shredding. The mixed inner leaflet content was gently pipetted on top of the outer leaflet phase. The tube was centrifuged at $2000 \times g$ for $3 \mathrm{~min}$. GUVs were washed and resuspended in glucose $(1 \mathrm{M})$. To check for PS in the inner leaflet, FITC-labeled recombinant lactadherin (rLactC2-FITC, $10 \mu \mathrm{g} / \mathrm{ml}$ ) was mixed with sucrose and overlaid with the inner leaflet lipid mix, following the protocol above. To monitor the level of PS in the outer leaflet GUVs were incubated with rLactC2FITC $(10 \mu \mathrm{g} / \mathrm{ml})$. GUVs were incubated $\mathrm{rCPn} 0473(20 \mu \mathrm{g} / \mathrm{ml})$ in the presence of rLactC2 $(10 \mu \mathrm{g} / \mathrm{ml})$. PS translocation was determined via ImageJ analysis, by measuring the rLactC2-FITC signals associated with the GUVs. The median of the maximum intensity at three different positions on one GUV (up to 24 GUVs per experiment) was measured and compared to the background signal intensity. The LactC2-FITC signal intensity associated with GUVs in which PS was restricted to the inner leaflet was set to 1 .

Cellular PL externalization assays. Externalized PS in HEp-2 cells was detected by incubation with $0.6 \mu \mathrm{l}$ of annexin and $30 \mu \mathrm{l}$ annexin staining buffer $(0.1 \mathrm{M}$ HEPES pH 7.4, $4.4 \mathrm{~K} \mathrm{NaCl} 5 \mathrm{mM} \mathrm{CaCl}_{2}$ ), or with $30 \mu$ of the recombinant FITClabeled, PS-binding domain of lactadherin in PBS (LactC2, $100 \mu \mathrm{g} / \mathrm{ml}$ ) at $4{ }^{\circ} \mathrm{C}$ for 30 min prior to fixation with PFA. The phosphoinositides PI(4,5)P2 and PI(3)P were detected with $30 \mu \mathrm{l}$ of the recombinant FITC-labeled PIP-binding domain of PLC $\gamma$ and FYVE respectively. As a control, the PIP biosensors were incubated with PFA-fixed HEp-2 cells, which were permeabilized with saponin prior treatment to enable intracellular PIP-binding.

PL externalization after treatment with recombinant protein was analyzed as follows: Confluent HEp-2 cells were overlaid with $250 \mu \mathrm{l}$ of culture medium containing $100 \mu \mathrm{g} / \mathrm{ml}$ recombinant protein. Cells were incubated with the protein solution for different times at $37^{\circ} \mathrm{C}$, and analyzed for PL externalization with the respective lipid marker as described above. The amount of externalized PS was quantified by measuring the total FITC using Nikon NIS software.

PS externalization during Cpn infection was analyzed as follows: Confluent HEp-2 cells were overlaid with $250 \mu \mathrm{l}$ culture medium containing infectious EBs $(\mathrm{MOI}=10)$. To measure the effects of EBs coated with recombinant protein on PS externalization, EBs were added to $100 \mu \mathrm{l}$ of protein solution $(1 \mu \mathrm{M})$ and incubated for $30 \mathrm{~min}$ at $4^{\circ} \mathrm{C}$ prior to infection $(\mathrm{MOI}=10)$. To measure the effects of selected antibodies on PS externalization, EBs were added to $100 \mu \mathrm{l}$ of antiserum suspensions and incubated for $2 \mathrm{~h}$ at $4{ }^{\circ} \mathrm{C}$. Infection was allowed to proceed up to 2 $\mathrm{h}$ at $37^{\circ} \mathrm{C}$. PS externalization was analyzed with either Annexin-V Fluor or LactC2
Apoptosis assay. To induce apoptosis in human epithelial cells, confluent HEp-2 cells were incubated in serum-free medium containing $2.5 \mu \mathrm{m}$ staurosporine at $37 \mathrm{C}$ for up to $16 \mathrm{~h}$. To test for induction of apoptosis by rCPn0473, cells were incubated with $100 \mu \mathrm{g} / \mathrm{ml}$ Protein for up to $16 \mathrm{~h}$. In order to analyze apoptosis early in infection, HEp-2 cells were infected with Cpn for $1 \mathrm{~h}$ at $37^{\circ} \mathrm{C}$. After extensive washing, cells were detached, pelleted $(5 \mathrm{~min}$ at $300 \times \mathrm{g}$ ) and resuspended in $100 \mu \mathrm{l}$ HBSS. Induction of apoptosis was analyzed by Western blotting using caspase-3and PARP-specific antibodies.

Reporting summary. Further information on research design is available in the Nature Research Reporting Summary linked to this article.

\section{Data availability}

The authors declare that the data supporting the findings of this study are available within the paper and its extended data files. Data underlying Figs. 1a, b, d, e, 2a, b, d, 3b, c, e, 4a, c, d, g, Supp. Figs. 1a, b, d, 4e are provided as Source Data files. All other data are available from the corresponding author upon reasonable requests.

Received: 7 August 2018; Accepted: 3 September 2019; Published online: 11 October 2019

\section{References}

1. Takatsu, H. et al. Phospholipid flippase activities and substrate specificities of human type IV P-type ATPases localized to the plasma membrane. J. Biol. Chem. 289, 33543-33556 (2014)

2. Hankins, H. M., Baldridge, R. D., Xu, P. \& Graham, T. R. Role of flippases, scramblases and transfer proteins in phosphatidylserine subcellular distribution. Traffic 16, 35-47 (2015).

3. Suzuki, J., Denning, D. P., Imanishi, E., Horvitz, H. R. \& Nagata, S. Xk-related protein 8 and CED-8 promote phosphatidylserine exposure in apoptotic cells. Science 341, 403-406 (2013).

4. Suzuki, J. et al. Calcium-dependent phospholipid scramblase activity of TMEM16 protein family members. J. Biol. Chem. 288, 13305-13316 (2013).

5. Grayston, J. T. et al. A new respiratory tract pathogen: Chlamydia pneumoniae strain TWAR. J. Infect. Dis. 161, 618-625 (1990).

6. Mölleken, K. \& Hegemann, J. H. The Chlamydia outer membrane protein $\mathrm{OmcB}$ is required for adhesion and exhibits biovar-specific differences in glycosaminoglycan binding. Mol. Microbiol. 67, 403-419 (2008).

7. Mölleken, K., Becker, E. \& Hegemann, J. H. The Chlamydia pneumoniae invasin protein Pmp21 recruits the EGF receptor for host cell entry. PLoS Pathog. 9, e1003325 (2013)

8. Mölleken, K. \& Hegemann, J. H. Acquisition of Rab11 and Rab11-Fip2-A novel strategy for Chlamydia pneumoniae early survival. PLoS Pathog. 13, e1006556 (2017).

9. Abdelrahman, Y. M. \& Belland, R. J. The chlamydial developmental cycle. FEMS Microbiol. Rev. 29, 949-959 (2005)

10. Fechtner, T., Galle, J. N. \& Hegemann, J. H. The novel chlamydial adhesin CPn0473 mediates the lipid raft-dependent uptake of Chlamydia pneumoniae. Cell Microbiol. 18, 1094-1105 (2016).

11. Fairn, G. D., Hermansson, M., Somerharju, P. \& Grinstein, S. Phosphatidylserine is polarized and required for proper $\mathrm{Cdc} 42$ localization and for development of cell polarity. Nat. Cell Biol. 13, 1424-1430 (2011).

12. Korhonen, J. T. et al. Chlamydia pneumoniae entry into epithelial cells by clathrin-independent endocytosis. Microb. Pathog. 52, 157-164 (2012).

13. Kuge, O., Nishijima, M. \& Akamatsu, Y. Phosphatidylserine biosynthesis in cultured Chinese hamster ovary cells. II. Isolation and characterization of phosphatidylserine auxotrophs. J. Biol. Chem. 261, 5790-5794 (1986)

14. van Meer, G., Voelker, D. R. \& Feigenson, G. W. Membrane lipids: where they are and how they behave. Nat. Rev. Mol. Cell Biol. 9, 112-124 (2008)

15. Goth, S. R. \& Stephens, R. S. Rapid, transient phosphatidylserine externalization induced in host cells by infection with Chlamydia spp. Infect. Immun. 69, 1109-1119 (2001).

16. Kay, J. G. \& Grinstein, S. Sensing phosphatidylserine in cellular membranes. Sens. (Basel) 11, 1744-1755 (2011).

17. Heyningen, S. V. Cholera toxin: interaction of subunits with ganglioside GM1 Science 183, 656-657 (1974).

18. Kyte, J. \& Doolittle, R. F. A simple method for displaying the hydropathic character of a protein. J. Mol. Biol. 157, 105-132 (1982).

19. Pautot, S., Frisken, B. J. \& Weitz, D. A. Engineering asymmetric vesicles. Proc. Natl. Acad. Sci. USA 100, 10718-10721 (2003). 
20. Elani, Y. et al. Measurements of the effect of membrane asymmetry on the mechanical properties of lipid bilayers. Chem. Commun. (Camb.) 51, 6976-6979 (2015).

21. Zaitseva, E. et al. Fusion stage of HIV-1 entry depends on virus-induced cell surface exposure of phosphatidylserine. Cell host microbe 22, 99-110 el17 (2017).

22. Murata-Kamiya, N., Kikuchi, K., Hayashi, T., Higashi, H. \& Hatakeyama, M. Helicobacter pylori exploits host membrane phosphatidylserine for delivery, localization, and pathophysiological action of the CagA oncoprotein. Cell Host Microbe 7, 399-411 (2010).

23. Semiramoth, N. et al. Afa/Dr-expressing, diffusely adhering Escherichia coli strain C1845 triggers F1845 fimbria-dependent phosphatidylserine externalization on neutrophil-like differentiated PLB-985 cells through an apoptosis-independent mechanism. Infect. Immun. 78, 2974-2983 (2010).

24. Hirama, T. et al. Membrane curvature induced by proximity of anionic phospholipids can initiate endocytosis. Nat. Commun. 8, 1393 (2017).

25. Mondal, M., Mesmin, B., Mukherjee, S. \& Maxfield, F. R. Sterols are mainly in the cytoplasmic leaflet of the plasma membrane and the endocytic recycling compartment in CHO cells. Mol. Biol. Cell 20, 581-588 (2009).

26. McMahon, H. T. \& Gallop, J. L. Membrane curvature and mechanisms of dynamic cell membrane remodelling. Nature 438, 590-596 (2005).

27. Kim, J. H., Jiang, S., Elwell, C. A. \& Engel, J. N. Chlamydia trachomatis Coopts the FGF2 signaling pathway to enhance infection. PLoS Pathog. 7, e1002285 (2011).

28. Römer, W. et al. Shiga toxin induces tubular membrane invaginations for its uptake into cells. Nature 450, 670-675 (2007).

29. Singh, P. K. et al. Dynein light chain 1 induces assembly of large Bim complexes on mitochondria that stabilize Mcl-1 and regulate apoptosis. Genes Dev. 31, 1754-1769 (2017).

\section{Acknowledgements}

We thank S. Birkelund (Aarhus University) and G. Zhong (University of Texas Health Science Center at San Antonio) for antibodies, J. Wiese (Heinrich-Heine-University Düsseldorf) for liposomes for pull-down experiments, S. Grinstein (University of Toronto) for the GST-Lactadherin-C2 expression vector, S. Wesselborg and C. Peters (Heinrich-Heine-University Düsseldorf) for the discussion of apoptosis and PS externalization, and for help with apoptosis-specific antibodies. We thank O. Kuge for the PS-deficient CHO cell line (PSA3). We are grateful to S. Hänsch (University of Düsseldorf) for providing us with the macro for microscopic analysis of the asymmetric GUVs with ImageJ software. We thank our reviewers for their constructive comments. J.N.G. was a scholarship holder funded by the Jürgen Manchot Foundation. T.F. was a scholarship holder of the Graduate School 'Molecules of Infection (MOI)' funded by the Jürgen Manchot Foundation. T.E. was supported by a Volkswagen Foundation grant (No. 92753) to Sven B. Gould. W.R. acknowledges support from the Excellence Initiative of the German Research Foundation (EXC 294), the
Ministry of Science, Research and the Arts of Baden-Württemberg (Az: 33-7532.20), and a Starting Grant from the European Research Council (Programme: "Ideas", ERC2011-StG 282105). This work was supported by the Deutsche Forschungsgemeinschaft, CRC 1208, project A05 to JHH.

\section{Author contributions}

T.F. identified LIPP. T.F. and J.N.G. designed, conducted, and evaluated the liposome and cell culture experiments. T.E., J.N.G., and W.R. designed and conducted GUV experiments. J.N.G. and J.H.H. wrote the manuscript with input from all co-authors. J.H.H. initiated and led the project and discussed experiments.

\section{Conflict of interest}

The authors declare no competing interests.

\section{Additional information}

Supplementary information is available for this paper at https://doi.org/10.1038/s41467019-12419-8.

Correspondence and requests for materials should be addressed to J.H.H.

Peer review information Nature Communications thanks Thomas Günther-Pomorski, Andreas Klos and the other, anonymous, reviewer(s) for their contribution to the peer review of this work. Peer reviewer reports are available.

Reprints and permission information is available at http://www.nature.com/reprints

Publisher's note Springer Nature remains neutral with regard to jurisdictional claims in published maps and institutional affiliations.

cC (i) Open Access This article is licensed under a Creative Commons Attribution 4.0 International License, which permits use, sharing, adaptation, distribution and reproduction in any medium or format, as long as you give appropriate credit to the original author(s) and the source, provide a link to the Creative Commons license, and indicate if changes were made. The images or other third party material in this article are included in the article's Creative Commons license, unless indicated otherwise in a credit line to the material. If material is not included in the article's Creative Commons license and your intended use is not permitted by statutory regulation or exceeds the permitted use, you will need to obtain permission directly from the copyright holder. To view a copy of this license, visit http://creativecommons.org/ licenses/by/4.0/.

(C) The Author(s) 2019 